\title{
Specific genomic DNA fragment analysis of different geographical clinical samples of shrimp white spot syndrome virus
}

\author{
Chu-Fang Lo ${ }^{1}$, Hui-Chen Hsu ${ }^{1}$, Meng-Feng Tsai ${ }^{1}$, Ching-Hui Ho ${ }^{1}$, Shao-En Peng ${ }^{1}$, \\ Guang-Hsiung Kou ${ }^{1}$, Donald V. Lightner ${ }^{2, *}$
}

\author{
${ }^{1}$ Department of Zoology, National Taiwan University, Taipei, Taiwan, ROC \\ ${ }^{2}$ Department of Veterinary Science, University of Arizona, Tucson, Arizona 85721, USA
}

\begin{abstract}
White spot syndrome (WSS) has been found in many species of shrimp and crabs, not just in Asia but globally. The causative agent is known as white spot syndrome virus (WSSV). In order to clarify the relatedness of WSSV from various geographic regions, we compared the viral DNA of a number of clinical samples of WSSV (1) China96-116A from Penaeus chinensis, (2) India95-314 from Penaeus monodon, (3) grocery store95-204 and grocery store96-115 from $P$. monodon possibly originating from Thailand, (4) crayfish $97-25$ from Orconectes punctimanus collected from the U.S. National Zoo, (5) Thailand95-46 from experimentally infected Penaeus vannamei, (6) South Carolina97-64 from P. vannamei, and (7) Texas95-242 and Texas96-7 from P. vannamei. These specimens were first examined by dot hybridization analysis with nucleic acid probes derived from a WSSV Taiwan isolate. Although the intensity of the hybridization signals varied, and although some specimens of India95314, crayfish97-25, Texas95-242 and Texas96-7 failed to give a detectable hybridization signal with certain probes, the broad consistency of dot hybridization data suggests that these WSSV clinical samples from different geographical locations are closely related. Following this analysis, all the specimens were examined using 10 virus-specific polymerase chain reactions (PCR). The amplification products were subsequently digested with Cfo I, Hae III, Hpa II and Rsa I restriction endonucleases to determine if there were any DNA fragment polymorphisms in the WSSV clinical samples. The results highlighted the genetic relatedness of all the WSSV clinical samples with the possible exception of a series of Texas viral samples which could be distinguished from the other geographic samples in some of the PCR-based tests
\end{abstract}

KEY WORDS: Genomic DNA analysis - Restriction profiles of specific viral DNA fragments - White spot syndrome virus WSSV.WSBV Geographical clinical samples

\section{INTRODUCTION}

The commercial cultivation of marine shrimp is now a global industry, and one of the biggest problems facing this industry is disease. White spot syndrome (WSS) is a viral disease which affects many commercially cultivated marine shrimp species, not just in Asia but globally (Lightner 1996, Flegel 1997). The principal clinical sign of WSS is the presence of white spots on the proximal surface of the cuticle of the diseased

\footnotetext{
- Addressee for correspondence.

E-mail: aquapath@ag.arizona.edu
}

shrimp. Affected individuals become lethargic and go off their feed. In shrimp ponds, they congregate in the shallows along the edges of the pond, and in culture tanks they sink inactively to the bottom, where they are frequently attacked and cannibalized by the healthier shrimp. WSS can cause up to $100 \%$ mortality (Chou et al. 1995, 1998), which leads to a correspondingly devastating economic impact. WSS has been formally recognized since 1992, but so far no significant resistance to this disease has been reported for any of the penaeid species (Lightner 1996).

The causative agent of WSS has, however, been identified: it is a non-occluded rod-shaped virus known 
as white spot syndrome virus (WSSV) or white spot 'baculovirus' (WSBV) (Wang et al. 1995, Wongteerasupaya et al. 1995, Lightner 1996, Lo et al. 1997). Although WSS has been observed in many species of shrimps and crabs from various geographic areas, for some time it has remained uncertain whether the causative agents of WSS are really identical or at least closely related, or whether, in fact, there are not several geographic strains or even several distinct viruses. Previous analysis has argued for a close relatedness for clinical samples of WSSV from different species of shrimps and crabs (Lo et al. 1996a, b), but all the tested specimens in these studies were collected from shrimp farms or coastal waters in the same geographic area of Taiwan. In the present study, therefore, to clarify the relatedness of different geographical clinical samples of WSSV, the viral DNA of a number of clinical samples of presumptive WSSV collected from Taiwan, China, India, Thailand, and the USA were compared using dot hybridization analysis with 11 distinct nucleic acid probes ( 3 to $12 \mathrm{kbp}$ ) derived from the inserts of plasmid clones selected from Sal I and Hind III genomic libraries of a Taiwan WSSV isolate from Penaeus monodon (Lo et al. 1996a).

Random amplification of polymorphic DNA (RAPD) is a widely used assay of DNA polymorphism in which RAPD profiles and/or markers distinguish between different geographic isolates and identify genetic variation within groups (J. G. Williams et al. 1990, 1992, Welsh \& McClelland 1990, C. L. Williams et al. 1994). To date, however, neither markers nor an RAPD assay have been developed for WSSV. In fact, currently there are almost no RAPD assay systems that are suitable for use with unpurified virus because of the possibility that the host DNA might influence the reaction. On the other hand, without an adequate in vitro culture system for shrimp viruses, plaque purification is impossible, so it is difficult to get a pure culture of each virus isolate. In the present study, therefore, specific DNA fragments were used instead for a restriction profile analysis of the unpurified WSSV clinical samples. The primers used to amplify specific DNA fragments and to investigate the restriction fragment length polymorphism (RFLP) were 20 to 25 nucleotides in length and based on the sequences derived from Taiwan WSSV DNA fragments from 9 distinct plasmid clones. Such long and specific primers usually generate an anticipated PCR product only if the clinical sample is specific to the primer.

\section{MATERIALS AND METHODS}

Virus. The geographic clinical samples of WSSV, all of which were from Dr Lightner's collection, were as follows: (1) China96-116A from Penaeus chinensis, (2) India95-314 from Penaeus monodon, (3) grocery store95-204 and grocery store96-115 from $P$. monodon possibly originating from Thailand, (4) crayfish97-25 from Orconectes punctimanus collected from the U.S. National Zoo, (5) Thailand95-46 from experimentally infected Penaeus vannamei, (6) South Carolina97-64 from P. vannamei, and (7) Texas95-242 and Texas96-7 from $P$. vannamei.

As we only discovered after the first battery of tests (see 'Results': Table 2), the original grocery store95-204 samples (which were somewhat degraded in storage) unexpectedly failed to hybridize with any of the probes. A second set of specimens (grocery store96-1.15) was therefore tested separately. Conversely, the South Carolina specimens showed hybridization patterns which were surprisingly similar to the Asian clinical samples. Two other specimens from the same South Carolina lot were therefore also subjected to a subsequent batch of tests along with 2 other American clinical samples, Texas95-242 and Texas96-7.

A WSSV Taiwan clinical sample (Lo et al. 1996a) was used as a positive control while WSSV-free shrimp tissues were used as negative controls throughout the experiment. DNA extracted from Baculovirus penaeiinfected (BP-infected) shrimp tissues was also included as a negative control in some of the dot hybridization analyses and WSSV diagnostic PCR (see 'Results': Table 2).

Extraction of viral DNA. A piece (approx. 100 to $200 \mathrm{mg}$ ) of tissue (gills, appendages, midgut, hepatopancreas or muscles) or an aliquot $(100 \mu l)$ of hemolymph from shrimp collected from various geographic areas was placed directly in a microfuge tube containing $1.2 \mathrm{ml}$ digestion buffer $(100 \mathrm{mM} \mathrm{NaCl}, 10 \mathrm{mM}$ Tris$\mathrm{HCl}, \mathrm{pH} 8,25$ mM EDTA, pH 8, 0.5\% N-lauryl sarcosine, $0.5 \mathrm{mg} \mathrm{ml}^{-1}$ proteinase $\mathrm{K}$ ) and crushed with a disposable stick. After $1 \mathrm{~h}$ incubation at $65^{\circ} \mathrm{C}, 600 \mu \mathrm{l}$ of the supernatant of the DNA/digestion buffer mixture was collected, and $100 \mu \mathrm{l}$ of $5 \mathrm{M} \mathrm{NaCl}$ and $70 \mu \mathrm{l}$ of CTAB/ $\mathrm{NaCl}$ stock solution $(10 \% \mathrm{~N}$-cetyl N,N,Ntrimethylammonium bromide in $0.7 \mathrm{M} \mathrm{NaCl}$ ) were then added. Following incubation at $65^{\circ} \mathrm{C}$ for $10 \mathrm{~min}$, the DNA was extracted with an equal volume of chloroform/isoamyl alcohol once, followed by an equal volume of phenol 2 to 3 times, and a double volume of chloroform/isoamyl alcohol once. The DNA was recovered by ethanol precipitation, dried and resuspended in $100 \mu$ of $0.1 \times$ TE buffer $(1 \times$ TE buffer: $10 \mathrm{mM}$ Tris$\mathrm{HCl}, 1 \mathrm{mM}$ EDTA, $\mathrm{pH} 7.6$ ) at $65^{\circ} \mathrm{C}$ for $30 \mathrm{~min}$, and then stored at $4^{\circ} \mathrm{C}$ until used for dot hybridization and PCR.

Detection of WSSV in collected shrimp samples by WSSV diagnostic PCR with the use of pms146F1/R1 primer set. A primer set derived from the sequence of a cloned WSSV Sal I 1461 bp DNA fragment (Lo et al. 
1996a) consisting of pms146F1 (5'-ACT ACT AAC TTC AGC CTA TCT AG-3') and pms146R1 (5'-TAA TGC GGG TGT AAT GTT CTT ACG A-3') was utilized for 1-step WSSV diagnostic PCR (Lo et al. 1996a) in order to confirm the presence of the virus in the collected shrimp samples (pms: Penaeus monodon WSSV Sal I fragment). The 1-step PCR was performed as follows. The DNA samples used for amplification totaled $0.1 \mu \mathrm{g}$ in a $100 \mu \mathrm{l}$ reaction mixture containing $10 \mathrm{mM}$ Tris$\mathrm{HCl}, \mathrm{pH} 8.8$ at $25^{\circ} \mathrm{C}, 50 \mathrm{mM} \mathrm{KCl}, 1.5 \mathrm{mM} \mathrm{MgCl}, 0.1 \%$ Triton X-100, $200 \mu \mathrm{M}$ of each dNTP, $100 \mathrm{pmol}$ of each primer, and 2 units of Taq DNA Polymerase (Life Technologies). The amplification was performed in a thermal cycler (Perkin-Elmer Corporation) for 1 cycle of $94^{\circ} \mathrm{C}$ for $4 \mathrm{~min}, 55^{\circ} \mathrm{C}$ for $1 \mathrm{~min}, 72^{\circ} \mathrm{C}$ for $2 \mathrm{~min}$, then 39 cycles of $94^{\circ} \mathrm{C}$ for $1 \mathrm{~min}, 55^{\circ} \mathrm{C}$ for $1 \mathrm{~min}, 72^{\circ} \mathrm{C}$ for $2 \mathrm{~min}$, plus a final $5 \mathrm{~min}$ extension at $72^{\circ} \mathrm{C}$ after 40 cycles. Control reactions containing no template DNA were run for all PCR reactions. A portion (10 $\mu \mathrm{l})$ from each of the completed $P C R$ reactions was mixed with $1 \mu l$ loading buffer and subjected to electrophoresis on $1 \%$ agarose gels containing ethidium bromide at a concentration of $0.5 \mu \mathrm{g} \mathrm{ml}^{-1}$, and visualized by ultraviolet transillumination.

As described previously (Lo et al. 1996a), the quality of the DNA extracted from the collected shrimp samples was checked with a primer set amplifying a decapod gene before the application of WSSV diagnostic PCR. For this purpose, 2 primers, 143F (5'- TGC CTT ATC AGC TNT CGA TTG TAG-3', where N represents G, A, T or C) and 145R (5'-TTC AGN TTT GCA ACC ATA CTT CCC-3'), derived from a highly conserved region of the $18 \mathrm{~S}$ rRNA sequence of decapods (Kim \& Abele 1990, Lo et al. 1996a) were used

Dot blot hybridization analysis. DNA samples were boiled for $10 \mathrm{~min}$ and then quenched on ice. An aliquot $(1 \mu \mathrm{l})$ of each of the DNA samples was dotted onto a sheet of positively charged nylon paper (Boehringer Mannheim, Mannheim, Germany) that had been presoaked with $5 \times \mathrm{SSC}(1 \times \mathrm{SSC}=150 \mathrm{mM} \mathrm{NaCl}, 15 \mathrm{mM}$ Sodium Citrate, $\mathrm{pH} 7.0$ ) for $5 \mathrm{~min}$ and air dried. After cross-linking the DNA with the membrane by UV light, the blot was used for hybridization with 11 WSSV probes which were non-radioactively labeled with digoxigenin (DIG)-dUTP (Boehringer Mannheim) by a random priming method. Following prehybridization at $65^{\circ} \mathrm{C}$ for $1 \mathrm{~h}$ in prehybridization solution (10 ml $5 \times$ SSC with $100 \mathrm{mg}$ of blocking reagent II, $50 \mu \mathrm{l}$ of $20 \%$ sarkosyl, $20 \mu \mathrm{l}$ of $10 \%$ SDS), the blot was hybridized at $65^{\circ} \mathrm{C}$ for $16 \mathrm{~h}$ with DIG-labeled probes. The detection of the DIG-labeled nucleotides in blots was accomplished by an immunological method using anti-digoxigenin antibody conjugated to alkaline phosphatase (Boehringer Mannheim) and CSPD ${ }^{\circledR}$, (Boehringer Mannheim) as a chemiluminescent substrate for alkaline phosphatase. The blot was exposed to Kodak X-OMAT film at room temperature for 1 to 10 min to record the chemiluminescent signal. In this study, the blots were reused several times for hybridization analysis with different probes. For reprobing, the blots were rinsed in water for $1 \mathrm{~min}$, treated with an alkaline solution $(0.2 \mathrm{~N} \mathrm{NaOH}, 1 \% \mathrm{SDS})$ at $37^{\circ} \mathrm{C}$ for $30 \mathrm{~min}$, and then rinsed in $2 \times \mathrm{SSC}$. Following the prehybridization, the blot was then hybridized with a new probe.

The inserts of 11 plasmid clones were used as probes: pms473 (12 kbp), pms484 (8 kbp), pms100 (6 kbp), pms321 (4.4 kbp), pms146 (1.5kbp), pmh2 (>9 kbp), pmh10 (>9 kbp), pmh11 (>9 kbp), pmh13 (>9 kbp), pmh32 (>9 kbp), pmh34 (>9 kbp). All these clones were selected from Sal I and Hind III genomic libraries of a Taiwan WSSV clinical sample (Lo et al. 1996a). The figures in parentheses indicate the size of the insert of each clone. The first set of DNA samples (1 to 47, see Table 2), i.e. the Asian and the first 2 South Carolina specimens, were analyzed with all 11 probes. Only 3 probes were used, however, for the second batch of DNA samples (48 to 71 , see Table 3 ), which included the new grocery store and South Carolina as well as the 2 Texas clinical samples.

Analysis of the DNA of WSSV geographical clinical samples by PCR with specific primer sets and restriction fragment length polymorphism. We used 10 primer pairs (Table 1) based on the DNA sequences (unpubl. data from Dr Guang-Hsiung Kou's laboratory) of 9 plasmid clones (pms54, pms94, pms98, pms120, pms146, pms321, pms473, pmh11, pmh13) selected from Sal I and Hind III genomic libraries of a Taiwan WSSV isolate from Penaeus monodon (Lo et al. 1996a). Ten amplicons, Apms54F1/R1 (0.96 kbp), Apms94F1/ R1 (0.94 kbp), Apms98F1/R1 (0.95 kbp), Apms120F1/ R1 (0.95 kbp), Apms146F1/R1 (1.5 kbp), Apms321F1/ R1 (0.9 kbp), Apms321F2/R2 (1.0 kbp), Apms473F3/R3 (1.1 kbp), Apmh11F1/R1 (1.0 kbp) and Apmh13F1/R1. $(1.2 \mathrm{kbp})$, were expected with 1 -step PCR and the primer sets. The size of each anticipated PCR product is indicated in parentheses. We used these 10 primer pairs and PCR to analyze the 71 DNA samples of various geographic WSSV clinical samples. To confirm that the amplified fragments were indeed virus specific, an additional internal primer set, pms98F2/R2 (Table 1), was also used to perform 2-step PCR. For Texas specimens, we also used Southern hybridization with probes prepared from PCR products of Taiwan WSSV isolate using internal primer sets of pms98 and pms 146 to detect WSSV-specific PCR products using a method described previously (Lo et al. 1996a, b). The Cfo I, Hae III, Hpa II, and Rsa I restriction profiles of some specific viral DNA fragments were also compared.

The thermal cycling program and reaction conditions for 1 -step PCR were the same as those described 
Table 1. PCR primer sequences used in this study

\begin{tabular}{|c|c|}
\hline Primer set & Primer sequence \\
\hline $1143 / 145$ & $\begin{array}{l}\text { 143F: 5'-TGCCTTATCAGCTNTCGATTGTAG-3' } \\
\text { 145R: 5'-TTCAGNTTTGCAACCATACTTCCC-3'd }\end{array}$ \\
\hline 2. pms54F1/R1 & $\begin{array}{l}\text { pms54F1: 5'-CGTAACAGGCTCGGTGCC-3' } \\
\text { pms54R1:5'-CAGCACGGATACGTTAAC-3' }\end{array}$ \\
\hline 3. $\mathrm{pms} 94 \mathrm{~F} 1 / \mathrm{R} 1$ & $\begin{array}{l}\text { pms94F1: 5'-CGGTCTCAGTAATTCGTC-3' } \\
\text { pms94R1: 5'-CCTCCATTTGCTGCAGTG-3' }\end{array}$ \\
\hline 4. $\mathrm{pms} 98 \mathrm{~F} 1 / \mathrm{R} 1$ & $\begin{array}{l}\text { pms98F1: 5'-GACAATGTTGGTATCGGTAG-3' } \\
\text { pms98R1: 5'-GAGCACGAGAAGCACGAC-3' }\end{array}$ \\
\hline 5. pms120F1/R1 & $\begin{array}{l}\text { pms120F1: 5'-GACATATACGCCACCAAGG-3' } \\
\text { pms120R1: 5'-GGCAGCGTCCATACTGTTC-3' }\end{array}$ \\
\hline 6. $\mathrm{pms} 146 \mathrm{~F} 1 / \mathrm{R} 1$ & $\begin{array}{l}\text { pmsI46F1. 5'-ACTACTAACTTCAGCCTATCTAG-3' } \\
\text { pms146R1: 5'-TAATGCGGGTGTAATGTTCTTACGA-3' }\end{array}$ \\
\hline 7. pms321-1F1/R1 & $\begin{array}{l}\text { pms321F1. 5'-CGCCACCAAGGAATTCGAAC-3' } \\
\text { pms321R1: 5'-GCAGACATGGCAGCGTCC-3' }\end{array}$ \\
\hline 8. pms321-2F1/R1 & $\begin{array}{l}\text { pms321F2: 5'-GCGAGCGGCGTACTACGAC-3' } \\
\text { pms321R2: 5'-GAGGCCACAGCCGAAGCTG-3' }\end{array}$ \\
\hline 9. $\mathrm{pms} 473 \mathrm{~F} 1 / \mathrm{R} 1$ & $\begin{array}{l}\text { pms473F3: 5'-AAGAGGAGGATTCTCCAGATCC-3' } \\
\text { pms473R3: 5'-CCAACACGGTACACGTAATTC-3' }\end{array}$ \\
\hline 10. pmh11F1/R1 & $\begin{array}{l}\text { pmh11F1: 5'-GGTGATTCTGCATCCAGC-3' } \\
\text { pmh11R1: 5'-GCGGATTCTATGAGGCGAG-3' }\end{array}$ \\
\hline 11. $\mathrm{pmh} 13 \mathrm{~F} 1 / \mathrm{R} 1$ & $\begin{array}{l}\text { pmh13F1: 5'-CAGGATGGTACAGAGGAC-3' } \\
\text { pmh13R1: 5'-GTCAATATAGCCATGGATGG-3' }\end{array}$ \\
\hline 12. $\mathrm{pms} 98 \mathrm{~F} 2 / \mathrm{R} 2$ & $\begin{array}{l}\text { pms98F2: 5'-CTGGGCCGTAAAGTAGTG-3' } \\
\text { pms98R2: 5'-CTGGACAATGCATGATGAG-3' }\end{array}$ \\
\hline${ }^{\mathrm{A}} \mathrm{N}$ represents $\mathrm{G}$, & T or $\mathrm{C}$ \\
\hline
\end{tabular}

shown in Table 2, only the 3 grocery store95-204 specimens consistently gave negative results in 1-step WSSV diagnostic PCR. As shown in Table 3, however, 10 of the Texas specimens gave negative results, and only 1 Texas specimen was positive in 1-step WSSV diagnostic PCR.

\section{Dot blot hybridization analysis}

In Table 2, apart from grocery store95-204, only India95-314\#3 and crayfish97-25\#1 failed to hybridize with all 11 WSSV probes, although the intensity of the hybridization signals varied. The broad consistency of these data suggests that these WSSV clinical samples from different geographical locations are closely related.

In the subsequent analysis of South Carolina and Texas specimens, 4 of 10 Texas specimens failed to give a positive hybridization signal with the pms146 and pms321 probes, while none of the Texas samples hybridized detectably with the pmh11 probe

above for WSSV diagnostic PCR using the pms146F1/ $\mathrm{R} 1$ primer set. For 1 -step PCR, $10 \mu \mathrm{l}$ of the 1 -step PCR reaction mixture was added to $90 \mu$ of PCR cocktail containing the inner primer pair, and this was then subjected to a second step of amplification in a thermal cycler (Perkin-Elmer Corporation) for 1 cycle of $94^{\circ} \mathrm{C}$ for $4 \mathrm{~min}, 55^{\circ} \mathrm{C}$ for $1 \mathrm{~min}, 72^{\circ} \mathrm{C}$ for $2 \mathrm{~min}$, then 39 cycles of $94^{\circ} \mathrm{C}$ for $1 \mathrm{~min}, 55^{\circ} \mathrm{C}$ for $1 \mathrm{~min}, 72^{\circ} \mathrm{C}$ for $2 \mathrm{~min}$, plus a final 5 min extension at $72^{\circ} \mathrm{C}$ after 40 cycles. Control reactions containing no template DNA were run for all $\mathrm{PCR}$ reactions. A portion (10 $\mu \mathrm{l})$ from each of the completed PCR reactions was mixed with $1 \mu$ loading buffer and subjected to electrophoresis on $1 \%$ agarose gels containing ethidium bromide at a concentration of $0.5 \mu \mathrm{g} \mathrm{ml}^{-1}$, and visualized by ultraviolet transillumination

\section{RESULTS}

\section{Detection of WSSV in collected shrimp samples by WSSV diagnostic PCR with the use of pms146F1/R1 primer set}

All the prepared templates were amplifiable when assessed by the shrimp DNA-specific primer set $143 / 145$ (Tables $2 \& 3$ ). Of the 47 tested DNA samples
(Table 3). Examples of the dot hybridization results are shown in Fig. 1.

\section{Analysis of the DNA of WSSV geographical clinical samples by PCR with specific primer sets and restriction fragment length polymorphism}

As predicted, the sizes of the 10 amplicons were very close, ranging from 0.9 to $1.5 \mathrm{kbp}$ (Fig. 2), but their restriction profiles varied (Fig. 3). We used these primer pairs to amplify DNA fragments from 71 DNA samples from 7 geographical clinical samples as indicated in Tables $2 \& 3$. Using the 10 primer pairs and PCR, 10 specific DNA fragments were yielded from the 47 tested DNA samples of 6 WSSV geographical clinical samples (China96-116A, India95-314, crayfish97-25, Thailand95-46, South Carolina97-64, grocery store 96-115). The sizes of major PCR products from 6 WSSV geographical clinical samples using the same primer pair were very similar and showed the expected sizes (Fig. 4). Unlike the other 6 geographical clinical samples, however, only 1 of the 16 DNA samples prepared from the 11 shrimp specimens of the 2 Texas clinical samples yielded PCR products with all 10 primer sets (Texas95-242-J60; Fig. 4, Lane 56). The Cfo I, Hae III, Hpa II, Rsa I RFLP profiles of the PCR 
Table 2. Analysis of WSSV geographical strains by dot blot hybridization with 11 WSSV probes derived from Taiwan strain. Numbers of plus symbols (i.e. between 1 and 4 ) corresponds to increasing levels of hybridization intensity on an arbitrary scale. (-) no hybridization. DNA sample names show collection area/individual shrimp no./tissue source for DNA extraction (G: gill; HP: hepatopancreas; Pl: pleopod; MG: midgut; M: muscle; H: hemolymph; Pe: pereiopod); HP/BP: homogenate of hepatopancreas of shrimp infected with Baculovirus penaei. Positive control: WSSV Taiwan clinical sample. Negative control: DNA from WSSV-free Penaeus monodon. For probes pms: Penaeus monodon WSSV Sal I fragment, and pmh: P. monodon WSSV Hind III fragment

\begin{tabular}{|c|c|c|c|c|c|c|c|c|c|c|c|c|c|}
\hline \multirow{2}{*}{$\begin{array}{l}\text { DNA samples } \\
\text { number and name }\end{array}$} & \multicolumn{11}{|c|}{ Probes } & \multicolumn{2}{|r|}{ PCR } \\
\hline & $\hat{E}^{3}$ & $5^{\infty}$ & $\hat{s}^{\circ}$ & $\hat{ह}^{2}$ & $5^{5}$ & है & हैं & $\hat{E}$ & $\stackrel{\vec{\xi}}{\vec{E}}$ & है & ह & $\mathrm{s}^{\xi^{2}}$ & $\overrightarrow{5}$ \\
\hline 1. China96-116A/\#1/G & ++ & t+ & + & + & + & ++ & +++ & t & ++ & +++ & t+ & +++ & t++ \\
\hline 2. China96-116A/\#1/HP & +++ & + & + & + & + & + & +++ & + & ++ & ++ & + & ++ & + \\
\hline 3. China96-116A/\#1/HP & +++ & + & + & + & + & + & +++ & + & ++ & ++ & + & +++ & +++ \\
\hline 4. China96-116A/\#1/HP & +++ & + & + & + & + & + & +++ & + & ++ & ++ & + & +++ & +++ \\
\hline 5. China96-116A//1/Pl & ++ & + & + & + & + & ++ & +++ & + & ++ & ++ & + & +++ & +++ \\
\hline 6. China96-116A/\#2/MG & +++ & + & + & + & + & ++ & +++ & + & ++ & ++ & + & +++ & +++ \\
\hline 7. China96-116A/\#2/G & ++ & + & + & + & + & ++ & +++ & + & ++ & ++ & + & +++ & +++ \\
\hline 8. China96-116A/\#3/MG & ++++ & +++ & t+ & ++ & t+ & $+t+$ & ++++ & + & +++ & ++++ & +++ & +++ & +++ \\
\hline 9. China96-116A/\#3/Pl & ++++ & +++ & ++ & ++ & ++ & +++ & ++++ & +++ & +++ & ++++ & +++ & +++ & +++ \\
\hline 10. India95-314/\#1/G & +++ & +++ & ++ & ++ & ++ & +++ & ++++ & +++ & ++ & +++ & +++ & +++ & +++ \\
\hline 11. India95-314/\#1/HP & +++ & +++ & ++ & ++ & ++ & +++ & ++++ & +++ & ++ & +++ & +++ & +++ & +++ \\
\hline 12. India95-314/\#1/Pl & +++ & +++ & ++ & ++ & ++ & +++ & ++++ & +++ & ++ & +++ & +++ & +++ & +++ \\
\hline 13. India95-314/ $\# 2 / G$ & ++ & +++ & ++ & +++ & ++ & $+t+$ & ++++ & +++ & ++ & +++ & +++ & +++ & +++ \\
\hline 14. India95-314/\#2/HP & ++ & +++ & ++ & $+t$ & ++ & +++ & ++++ & +++ & ++ & +++ & +++ & +++ & +++ \\
\hline 15. India95-314/\#2/Pl & +++ & +++ & ++ & +++ & ++ & +++ & ++++ & +++ & ++ & +++ & +++ & +++ & +++ \\
\hline 16. India95-314/\#3/G & - & + & - & - & - & + & - & - & - & + & - & +++ & ++ \\
\hline 17. India95-314/\#3/HP & - & - & + & - & - & - & - & - & - & - & - & +++ & + \\
\hline 18. India95-314/\#3/Pl & - & - & + & - & - & - & + & - & - & - & - & +++ & + \\
\hline 19. Grocery store95-204/\#1/M & - & - & - & - & - & - & - & - & - & - & - & +++ & - \\
\hline 20. Grocery store95-204/\#1/M & - & - & - & - & - & - & - & - & - & - & - & +++ & - \\
\hline 21. Grocery store95-204/\#2/M & - & - & - & - & - & - & - & - & - & - & - & +++ & - \\
\hline 22. Grocery store95-204/\#2/M & - & - & - & - & - & - & - & - & - & - & - & +++ & - \\
\hline 23. Grocery store95-204/\#3/M & - & - & - & - & - & - & - & - & - & - & - & +++ & - \\
\hline 24. Grocery store95-204/\#3/M & - & - & - & - & - & - & - & - & - & - & - & +++ & - \\
\hline 25. Crayfish97-25/\#1/G & - & + & - & + & - & + . & ++ & + & + & + & + & +++ & ++ \\
\hline 26. Crayfish97-25/\#1/Pl & - & + & - & + & - & + & ++ & + & + & + & + & +++ & ++ \\
\hline 27. Crayfish97-25/\#2/G & ++ & +++ & ++ & ++ & ++ & +++ & +++ & +++ & +++ & ++ & ++ & +++ & +++ \\
\hline 28. Crayfish97-25/\#2/Pl & $+t$ & +++ & ++ & ++ & $+t$ & +++ & +++ & +++ & +++ & ++ & +++ & +++ & +++ \\
\hline 29. Crayfish97-25/\#3/G & +++ & ++ & $+t$ & ++ & ++ & +++ & +++ & +++ & +++ & ++ & +++ & $++t$ & +++ \\
\hline 30. Crayfish97-25/\#3/Pl & ++++ & $+t+$ & ++ & +++ & ++ & +++ & ++++ & +++ & ++++ & ++++ & +++ & +++ & +++ \\
\hline 31. Thailand95-46/\#1/B1 & + & + & - & + & - & + & ++ & + & + & + & + & + & ++ \\
\hline 32. Thailand95-46/\#1/B2 ${ }^{\mathrm{a}}$ & + & + & + & + & - & + & ++ & + & + & + & + & ++ & ++ \\
\hline 33. Thailand95-46/\#1/B3 ${ }^{\circ}$ & + & + & - & + & - & + & ++ & + & + & + & + & ++ & +++ \\
\hline 34. Thailand95-46/井1/B4a & + & + & + & + & + & + & ++ & + & + & + & + & ++ & +++ \\
\hline 35. South Carolina97-64/\#1/H & ++ & ++ & + & + & t+ & ++ & ++ & ++ & ++ & ++ & ++ & ++ & +++ \\
\hline 36. South Carolina97-64/\#2/G & +++ & +++ & ++ & ++ & ++ & $t+t$ & +++ & +++ & +++ & +++ & $+t+$ & +++ & $+t+$ \\
\hline 37. South Carolina97-64/\#2/G & +++ & +++ & ++ & ++ & ++ & +++ & +++ & +++ & +++ & +++ & +++ & +++ & +++ \\
\hline 38. South Carolina97-64/\#2/G & +++ & +++ & ++ & +++ & ++ & +++ & +++ & +++ & +++ & +++ & ++++ & +++ & +++ \\
\hline 39. South Carolina97-64/\#2/Pe & ++ & +++ & ++ & ++ & ++ & +++ & +++ & +++ & +++ & +++ & +++ & +++ & +++ \\
\hline 40. South Carolina97-64/\#2/Pe & ++ & +++ & + & + & ++ & ++ & +++ & +++ & +++ & +++ & ++ & +++ & +++ \\
\hline 41. South Carolina97-64/\#2/Pe & ++ & +++ & + & ++ & ++ & +++ & +++ & +++ & +++ & +++ & +++ & +++ & +++ \\
\hline 42. South Carolina97-64/\#2/Pl & ++ & +++ & ++ & ++ & ++ & +++ & +++ & +++ & +++ & +++ & +++ & +++ & +++ \\
\hline 43. South Carolina97-64/\#2/PI & +++ & +++ & ++ & ++ & ++ & +++ & +++ & +++ & +++ & +++ & +++ & +++ & +++ \\
\hline 44. South Carolina97-64/\#2/PI & +++ & +++ & $+t$ & ++ & ++ & +++ & +++ & +++ & +++ & +++ & +++ & +++ & +++ \\
\hline 45. South Carolina97-64/\#2/M & ++ & +++ & ++ & ++ & ++ & +++ & +++ & +++ & +++ & +++ & +++ & +++ & +++ \\
\hline 46. South Carolina97-64/\#2/M & ++ & $++t$ & ++ & ++ & ++ & +++ & +++ & +++ & +++ & +++ & +++ & +++ & +++ \\
\hline 47. South Carolina97-64/\#2/M & ++ & +++ & ++ & ++ & ++ & +++ & +++ & +++ & +++ & +++ & +++ & +++ & +++ \\
\hline $\mathrm{HP} / \mathrm{BP}$ & - & - & - & - & - & - & - & - & - & - & - & + & - \\
\hline Positive control & +++ & ++ & ++ & ++ & ++ & +++ & +++ & +++ & +++ & +++ & +++ & +++ & +++ \\
\hline Negative control & - & - & - & - & - & - & - & - & - & - & - & +++ & - \\
\hline
\end{tabular}


Table 3. Analysis of WSSV geographical strains by dot blot hybridization with 11 WSSV probes derived from Taiwan strain. Number of plus symbols (i.e between 1 and 4) corresponds to increasing level of hybridization intensity on an arbitrary scale. N.D. test not done. (-) no hybridization. DNA sample names show collection area/individual shrimp no./tissue sources for DNA extraction (G: gill; Pl: pleopod, M: muscle). Positive control: WSSV Taiwan clinical sample. Negative control: DNA from WSSV-free Penaeus monodon

\begin{tabular}{|c|c|c|c|c|c|c|c|c|c|c|c|c|c|}
\hline \multirow{2}{*}{$\begin{array}{l}\text { DNA samples } \\
\text { number and name }\end{array}$} & \multicolumn{11}{|c|}{ Probes } & \multicolumn{2}{|r|}{ PCR } \\
\hline & $\hat{s}^{\infty}$ & $\sum^{\infty}$ & $\frac{8}{5^{\circ}}$ & $\hat{\xi}^{\hat{\xi}}$ & $\xi^{\infty}$ & ह & $\stackrel{8}{E}$ & 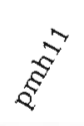 & $\stackrel{\vec{\xi}}{\xi}^{3}$ & 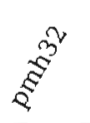 & $\sum^{\xi^{\infty}}$ & 今 & $\overrightarrow{5}$ \\
\hline 48. South Carolina97-64/\#3/G & N.D. & N.D. & N.D. & t+ & t+ & N.D. & N.D. & +t & N.D. & N.D. & N.D. & + & + \\
\hline 49. South Carolina97-64/\#3/Pl & N.D. & N.D. & N.D. & ++ & ++ & N.D. & N.D. & ++ & N.D. & N.D. & N.D. & + & + \\
\hline 50. South Carolina97-64/\#4/G & N.D. & N.D. & N.D. & ++ & ++ & N.D. & N.D. & ++ & N.D. & N.D. & N.D. & + & + \\
\hline 51. South Carolina97-64/\#4/PI & N.D. & N.D. & N.D. & ++ & ++ & N.D. & N.D. & ++ & N.D. & N.D. & N.D. & + & + \\
\hline 52. Grocery store96-115/\#4/Pl & N.D. & N.D. & N.D. & ++ & ++ & N.D. & N.D. & ++ & N.D & N.D. & N.D. & + & + \\
\hline 53. Grocery stare96-115/\#4/M & N.D. & N.D. & N.D. & ++ & ++ & N.D. & N.D. & ++ & N.D. & N.D. & N.D. & + & + \\
\hline 54. Grocery store96-115/\#5/Pl & N.D. & N.D. & N.D. & ++ & ++ & N.D. & N.D. & ++ & N.D. & N.D. & N.D. & + & + \\
\hline 55. Grocery store96-115/\#5/M & N.D. & N.D. & N.D. & ++ & ++ & N.D. & N.D. & t+ & N.D. & N.D. & N.D. & + & + \\
\hline 56. Texas95-242-J60/\#1/M & N.D. & N.D. & iv.D. & - & - & N.D. & N.D. & - & N.D. & N.O. & N.D. & + & + \\
\hline 57. Texas95-242-J61/\#2/M & N.D. & N.D. & N.D. & - & - & N.D. & N.D. & - & N.D. & N.D. & N.D. & + & - \\
\hline 58. Texas95-242-J43/\#3/M & N.D. & N.D. & N.D. & - & - & N.D. & N.D. & - & N.D. & N.D. & N.D. & + & - \\
\hline 59. Texas95-242-J43/\#4/M & N.D. & N.D. & N.D. & - & - & N.D. & N.D. & - & N.D. & N.D. & N.D. & + & - \\
\hline 60. Texas95-242-J43/\#5/M & N.D. & N.D. & N.D. & + & + & N.D. & N.D. & - & N.D. & N.D. & N.D. & + & - \\
\hline 61. Texas95-242-J43/\#6/M & N.D. & N.D. & N.D. & + & + & N.D. & N.D. & - & N.D. & N.D. & N.D. & + & - \\
\hline 62. Texas96-7/\#7/G & N.D. & N.D. & N.D. & + & + & N.D. & N.D. & - & N.D. & N.D. & N.D. & + & - \\
\hline 63. Texas96-7/\#7/Pl & N.D. & N.D. & N.D. & + & + & N.D. & N.D. & - & N.D. & N.D. & N.D. & + & - \\
\hline 64. Texas96-7/\#8/G & N.D. & N.D. & N.D. & + & + & N.D. & N.D. & - & N.D. & N.D. & N.D. & + & - \\
\hline 65. Texas $96-7 / \# 8 / \mathrm{Pl}$ & N.D. & N.D. & N.D. & + & + & N.D. & N.D. & - & N.D. & N.D. & N.D. & + & - \\
\hline 66. Texas $96-7 / \# 9 / G$ & N.D. & N.D. & N.D. & + & + & N.D. & N.D. & - & N.D. & N.D. & N.D. & + & - \\
\hline 67. Texas96-7/\#9/Pl & N.D. & N.D. & N.D. & + & + & N.D. & N.D. & - & N.D. & N.D. & N.D. & + & - \\
\hline 68. Texas $96-7 / \# 10 / G$ & N.D. & N.D. & N.D. & + & + & N.D. & N.D. & - & N.D. & N.D. & N.D. & + & - \\
\hline 69. Texas96-7/\#10/Pl & N.D. & N.D. & N.D. & + & + & N.D. & N.D. & - & N.D. & N.D. & N.D. & + & - \\
\hline 70. Texas96-7/\#11/G & N.D. & N.D. & N.D. & + & + & N.D. & N.D. & - & N.D. & N.D. & N.D. & + & - \\
\hline 71. Texas96-7/\#11/P1 & N.D. & N.D. & N.D. & + & + & N.D. & N.D. & - & N.D. & N.D. & N.D. & + & - \\
\hline Positive control & & & & ++ & ++ & & & +++ & & & & + & - \\
\hline Negative control & & & & - & - & & & - & & & & + & - \\
\hline
\end{tabular}

products yielded by this Texas clinical sample suggest that it is in fact very similar if not identical to the other 6 WSSV geographic clinical samples. One of these RFLP profile comparisons is shown in Fig. 5. The other Texas specimens were 1-step PCR negative with most of the primer sets (Fig. 4, Lanes 57 to 71), although some of them did yield PCR products in the tests with pmh11F1/R1, pmh13F1/R1, pms54F1/R1, pms94F1/R1, pms98F1/R1, pms120F1/R1, and pms321 F2/R2. Interestingly, the PCR products yielded by the Texas95-242 specimens were always of the anticipated size (Fig. 4, Lanes 56 to 61), while the faint PCR products yielded by the Texas $96-7$ specimens in the tests with primer sets pmh11FR1/R1, pms94F1/R1, pms98F1/R1 and pms321F2/R2 were all of unexpected sizes (Fig. 4, Lanes 62 to 71 ). When the internal primer set 98F2/R2 was used to do 2-step PCR, however, all the Texas samples (Lanes 56 to 71 ) yielded bands of the expected size that were similar to the other 6 geographic clinical samples (Fig. 4). However, the intensity of 2-step PCR product bands yielded by Texas samples (Lanes 57 to 71 ) was much weaker than 1 Texas sample (Lane 56) and the other 6 geographic clinical samples (Fig. 4), this implies that the amount of virus in Texas samples (57 to 71) was much less than in the other clinical samples. Southern hybridization analysis also revealed that bands of expected size were present in 1-step PCR products of most of the Texas clinical samples (Fig. 6), thereby indicating the existence of WSSV in these specimens. Even so, there were some anomalies: some of the major PCR products of the Texas96-7 specimens (Fig. 6, Lanes 62 to 71) were visible in the Apms98F1/ R1 agarose gel but had a smaller than expected size and failed to hybridize with the Apms98F2/R2 probe, while those bands that successfully hybridized with the Apms98F2/R2 probe had the anticipated size, but were invisible in the Apms98F1/R1 agarose gel. 
A

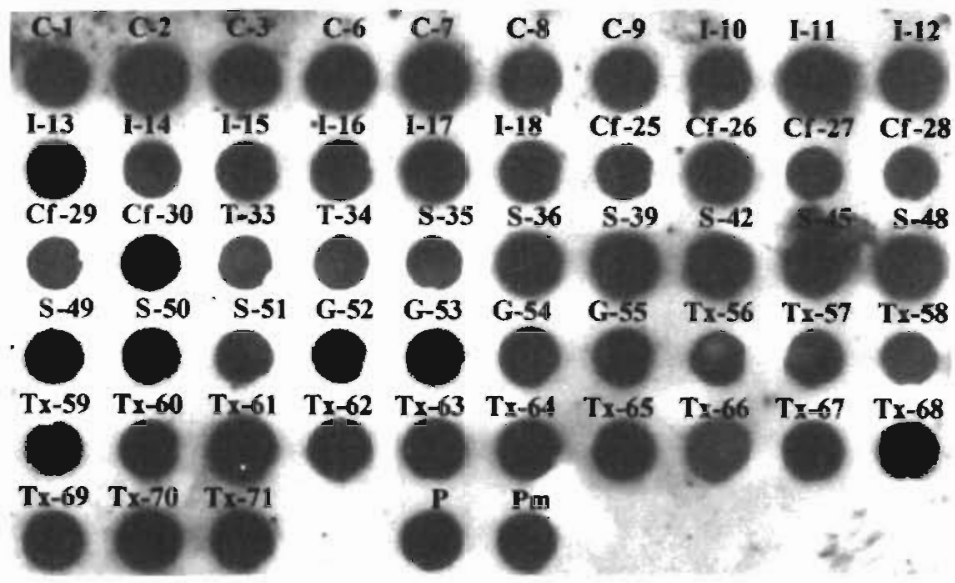

B

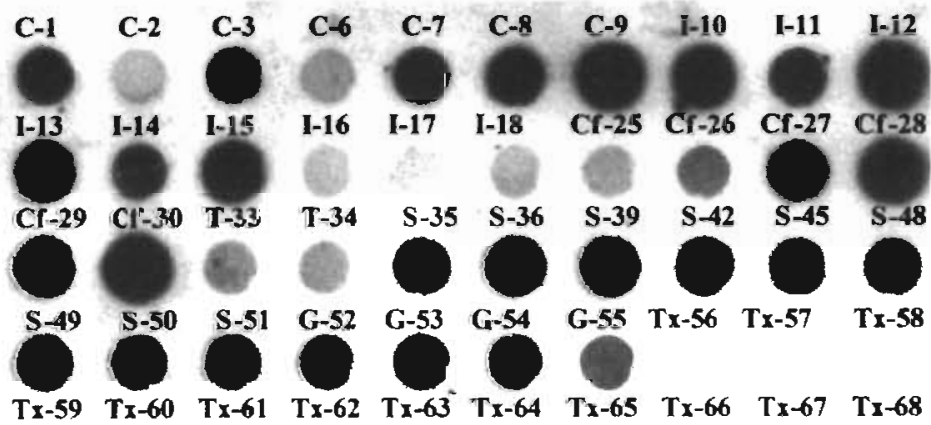

$T x-69 T x-70 \quad T x-71$

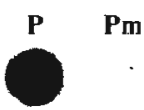

Fig.1. Dot hybridization of WSSV geographical clinical samples with (A) shrimp 18 S rRNA gene probe and (B) WSSV probe derived from the amplicon Apms146F1/R1. The geographic origin of each DNA sample is indicated above each dot $(\mathrm{C}$ : China; I: India; $\mathrm{Cf}$ : Crayfish; $T$ : Thailand; $\mathrm{S}$ : South Carolina; $\mathrm{G}$ : grocery store; Tx: Texas), and the DNA sample numbers correspond to those used in Tables 2 \& 3. P. Taiwan clinical sample. Pm: healthy Penaeus monodon

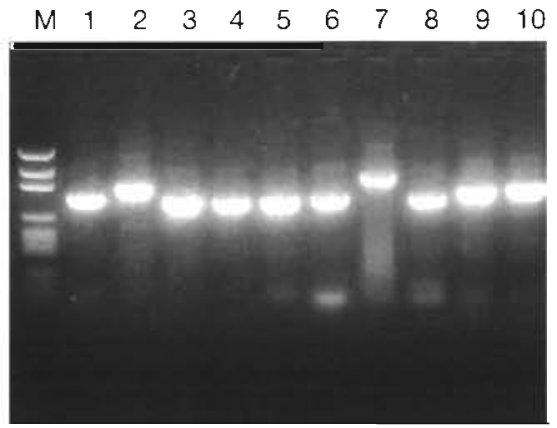

Fig. 2. Ten amplicons, Apmh11F1/R1 (Lane 1), Apmh13F1/R1 (Lane 2), Apms54F1/R1 (Lane 3), Apms94F1/R1 (Lane 4), Apms98F1/R1 (Lane 5), Apms120F1/R1 (Lane 6), Apms146 F1/R1 (Lane 7), Apms321F1/R1 (Lane 8), Apms321F2/R2 (Lane 9), and Apms473F3/R3 (Lane 10), amplified from WSSV Taiwan clinical sample. Lane M: pGEN DNA size marker
Cfo I restriction profile

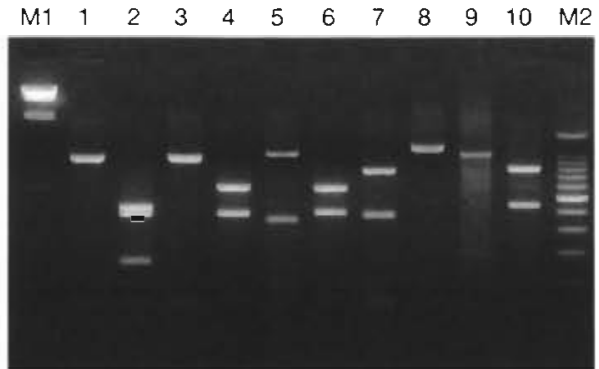

Rsa I restriction profile

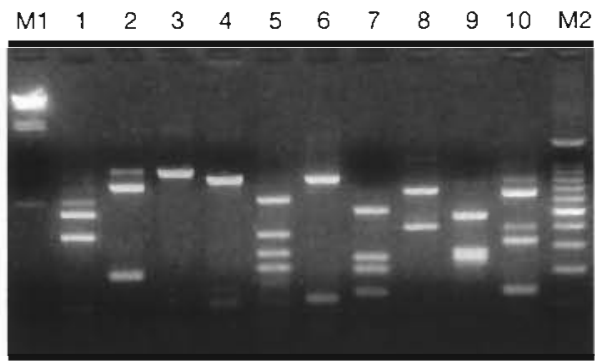

Hpa II restriction profile

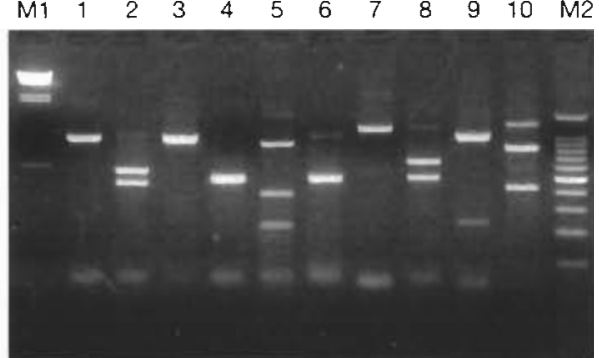

Hae III restriction profile

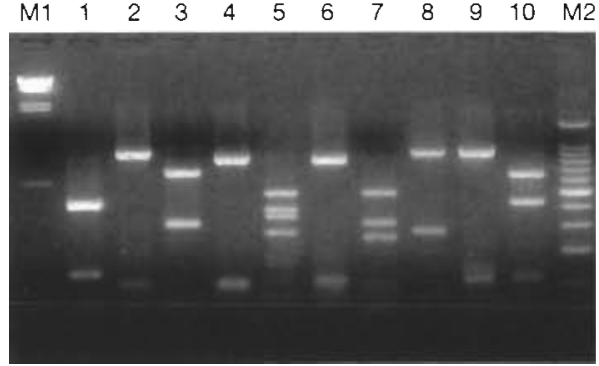

Fig. 3. Restriction profiles of 10 amplicons amplified from WSSV Taiwan clinical sample and cleared with cfo I, Rsa I, Hpa II, and Hae III nucleases. (Lane 1) Apms54F1/R1; (Lane 2) Apms94F1/R1; (Lane 3) Apms98F1/R1; (Lane 4) Apms120 F1/R1; (Lane 5) Apms146F1/R1; (Lane 6) Apms321F1/R1; (Lane 7) Apms321F2/R2; (Lane 8) Apms473F3/R3; (Lane 9) Apmh11F1/R1; (Lane 10) Apmh13F1/R1. M1: lambda phage

DNA Hind III fragments; M2: the $100 \mathrm{bp}$ DNA ladder

When cleaved with Cfo I, Hae III, Hpa II and Rsa I restriction endonucleases, the RFLPs of China96-116A, India95-314, crayfish97-25, Thailand95-46, South Carolina97-64, grocery store96-115 and Texas95-242 were very similar, suggesting again that all 7 of these 


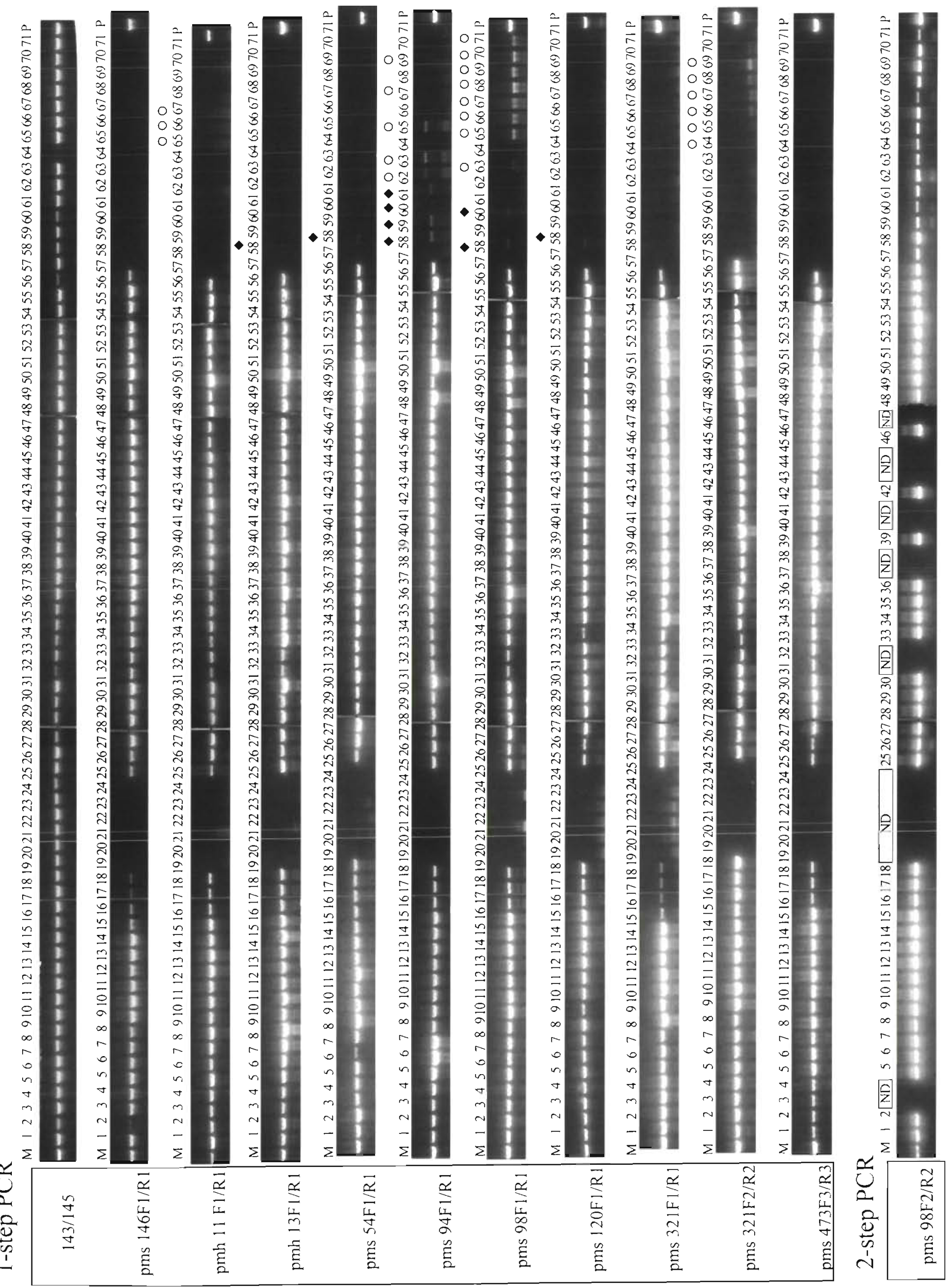


Fig. 4. Amplification of amplicons 143/145, Apms146F1/R1, Apmh11F1/R1, Apmh13F1/R1, Apms54F1/R1, Apms94F1/R1, Apms98 F1/R1, Apms120F1/R1, Apms321F1/R1, Apms321F2/R2, and Apms473F3/R3 by 1-step PCR and Apms98F2/R2 by 2-step PCR from the WSSV geographical clinical samples indicated in Tables 2 \& 3. (Lane number corresponds to DNA sample number.) M: PCR size marker. P: Taiwan WSSV. ( $\bullet$ Faint band with the expected size; (O) band with unexpected size. ND: not detected

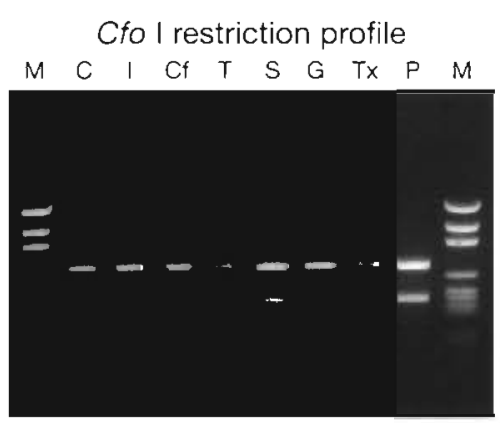

Rsa I restriction profile

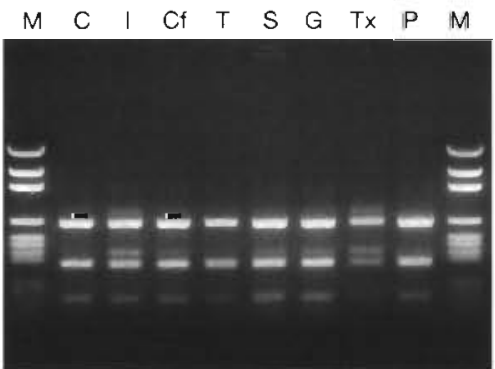

Hpa II restriction profile

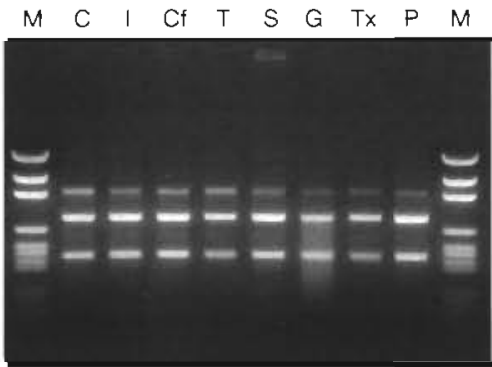

Hae III restriction profile
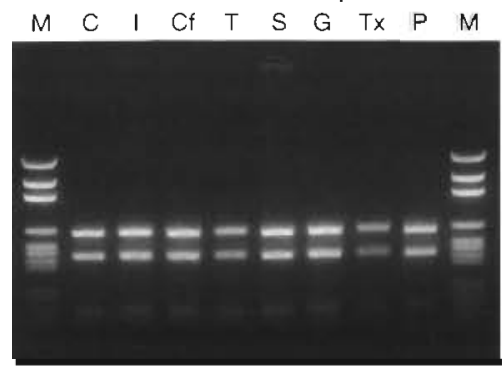

Fig. 5. Restriction profiles of Apmh13F1/R1 amplified from China96-116A. (Lane C), India95-314 (Lane 1), Crayfish97-25 (Lane Cf), Thailand95-46 (Lane T), South Carolina97-64 (Lane S), grocery store96-115 (Lane G); Texas95-242-J60 (Lane Tx) and Taiwan WSSV (Lane P) cleaved with Cfo I, Rsa I, Hpa II and

Hae III endonucleases. M: pGEM DNA marker

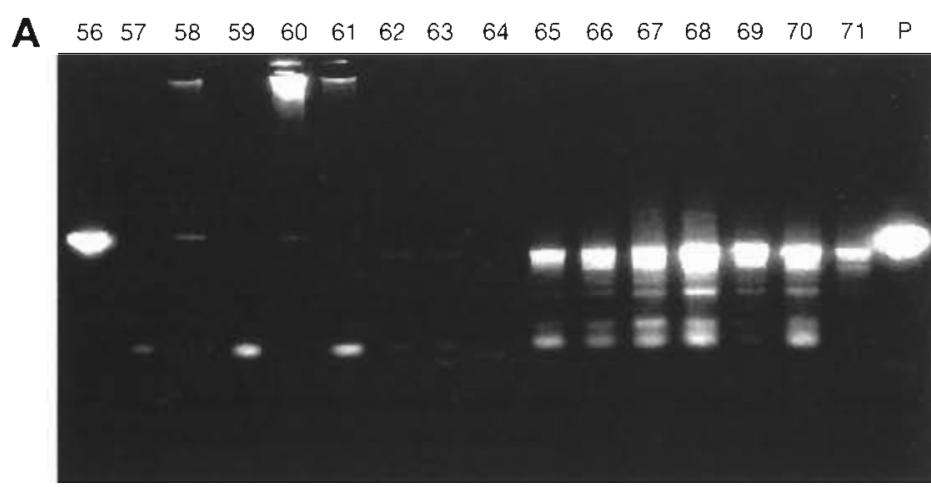

$\begin{array}{llllllllllllllllll}\text { В } & 56 & 57 & 58 & 59 & 60 & 61 & 62 & 63 & 64 & 65 & 66 & 67 & 68 & 69 & 70 & 71 & P\end{array}$
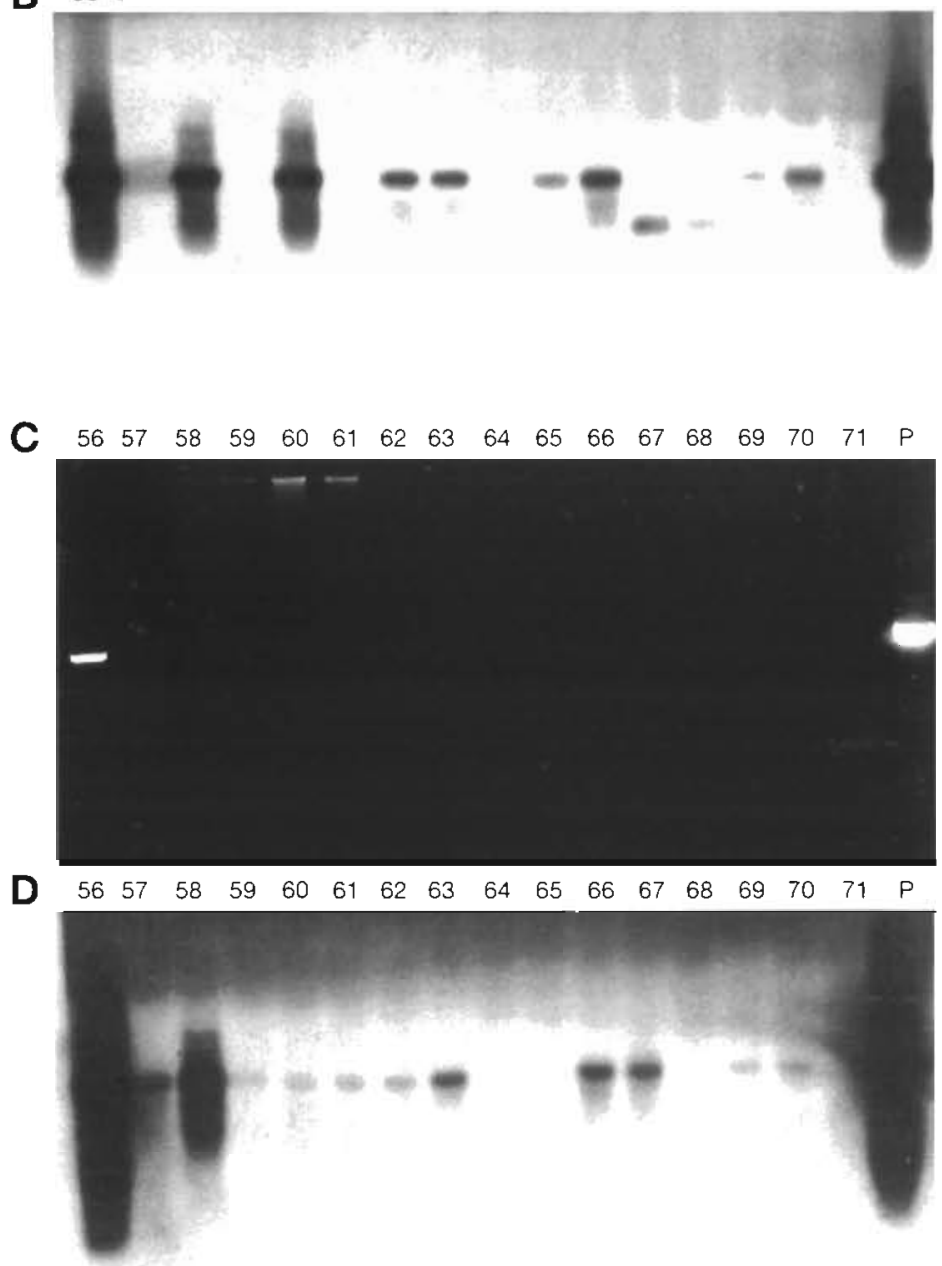

Fig. 6. Southern blot hybridization analysis of PCR products. Ethidium bromide-stained agarose gel of PCR products of Apms98F1/R1 (A) and Apms146F1/R1 (C) are Southern blot hybridized with Apms98 F2/R2 probe (B) and Apms146F2/R2 probe (D), respectively. Lane numbers correspond to the DNA sample numbers used in Table 3 
WSSV geographical clinical samples belong to the same virus (group). Examples of RFLP analysis are shown in Fig. 5.

\section{DISCUSSION}

As in previous papers, the causal agent of the disease or syndrome is referred to here as WSSV (white spot syndrome virus). However, as several viruses/ baculoviruses have been described from the host species investigated here, Penaeus monodon (Lightner 1996), it should be borne in mind that the possibility of simultaneous infection by one or more viruses can not be completely excluded in the present study. Accordingly, since we did not purify the virus from the diseased shrimp, the analyzed specimens were therefore termed 'clinical samples' rather than 'virus isolates'. In order that the impurity of the specimens might not lead to difficulties in interpreting the results, the PCR primers used here were derived from the genomic DNA extracted from purified virions of a WSSV Taiwan isolate. These primer sequences (Table 1), and even the fragment sequences from which they were derived, are unique. (unpubl. data from Dr GuangHsiung Kou's laboratory). These highly specific primers thus minimize the possibility of any interference that might result from a mixed viral infection.

In the dot hybridization analysis, India95-314\#3 and crayfish97-25\#1 (Table 2) failed to hybridize with some probes. These anomalous results might have been caused by the virus content in these specimens being very low. Alternatively, some mutants may have existed in shrimp collected from the same geographical location or even from the same culture farm. The PCR tests using 10 primer sets (Fig, 4, Lanes 16 to 18 , 25 , and 26), however, suggest that the virus in these specimens is similar to other geographic clinical samples (e.g. China96-116, Thailand95-46, South Carolina97-64), so that a low virus content in the samples is very likely to have been the cause of the failure in the hybridization tests.

As shown in Fig. 4, the PCR tests using the 10 primer sets derived from a WSSV Taiwan isolate provided clear evidence that all the specimens of China96-116A, India95-314, crayfish97-25, Thailand95-46, South Carolina97-64, and grocery store96-115 were infected with WSSV or a WSSV-like virus. Almost all the amplicons of the 10 primer sets from these DNA samples appeared as a single band with the expected sizes. Even though a few primer sets gave rise to PCR products from WSSV negative DNA samples (e.g. grocery store95-204 which were somewhat degraded in storage) as either multiple bands (e.g. pms321F1/R1) or single bands of unexpected size (e.g. pms98F1/R1), the results as a whole not only demonstrate the similarity of these clinical samples but also show that these clinical samples can be easily detected using any of the 10 primer sets used for the present study.

The generally good yields seen in Fig. 4 suggest that all the primer sets annealed successfully with the DNA from 48 (DNA samples 1 to 18 and 25 to 55) of WSSV clinical samples from 6 geographical areas (China 96-116A, India95-314, crayfish97-25, Thailand95-46, South Carolina97-64, grocery store96-115). This further suggests that there is very little sequence variation at least within the primer regions of these 6 WSSV geographical clinical samples. Furthermore, the 10 primer sets used in this study correspond to 10 entirely different DNA fragments of the entire WSSV genome. These fragments, of approximately $1 \mathrm{kbp}$ each, therefore constitute a reasonable sampling of the complete genome, so that, since the sizes of the major PCR products from all 6 WSSV geographical clinical samples using the same primer pair were very similar (Fig. 4), it can be argued that substantial similarity exists in the genome structure as a whole.

For 2 Texas clinical samples, however, unexpected reaction patterns were found: although the bands shown by the Texas95-242 specimens were of the expected size, these bands were faint at best and, in many of the PCR tests, no PCR product was yielded at all (Fig. 4, Lanes 57 to 61). We therefore speculate that the virus in Texas95-242 specimens may well be different from the Asian WSSV clinical samples. Further sequence analysis will be required to confirm or refute this hypothesis.

Texas96-7 specimens, on the other hand, yielded faint PCR products in the tests with primer sets pmh11F1/R1, pms94F1/R1, pms98F1/R1 and pms321 F2/R2 but all of them had unexpected sizes (Fig. 4, Lanes 62 to 71 ). With 2-step PCR (Fig. 4) and Southern hybridization (Fig. 6), however, we found at least some bands with expected sizes in all of the Texas specimens. Although this suggests that the Texas specimens were all infected by WSSV or a WSSV-like virus, the evidence of the PCR reaction patterns argues that the major virus population of Texas WSSV might not be identical to the other geographic clinical samples. In particular, the unexpectedly small size of the major Apms98F1/R1 product yielded by the Texas96-7 specimens (Fig. 6, Lanes 62 to 71 ) and its failure to hybridize with the Apms98F2/R2 probe suggest this amplicon has little sequence homology to the expected Apms98 F1/R1 PCR product. Before further study can resolve this question, however, the virus will need to be purified. PCR analysis could then check whether the PCR products of unexpected size still exist. If these anomalous amplicons, were still found, we would expect DNA sequencing to reveal a low homology with the 
Asian isolates and that the genomic restriction profiles would be distinct. This work would be time consuming, but we believe it is important for the anomalies reported here to be properly accounted for.

Acknowledgements. This work was supported by the National Science Council Grant no. NSC86-2311-B-002-051 and Grant no. NSC87-2311-B-002-070; Gulf Coast Research Laboratory Consortium Marine Shrimp Farming Program, CSREES, U. S. Dept. Agriculture Grant no. 95-38808-1424, National Sea Grant Program, U.S. Dept. of Commerce Grant no. NA56RG0617; and the National Fishery Institute. We are indebted to $\mathrm{Mr}$ Paul Barlow for his helpful criticism of the manuscript.

\section{LITERATURE CITED}

Chou HY, Huang CY, Wang CH, Chiang HC, Lo CF (1995) Pathogenicity of a baculovirus infection causing white spot syndrome in cultured penaeid shrimp in Taiwan. Dis Aquat Org 23:165-173

Chou HY, Huang CY, Lo CF, Kou GH (1998) Studies on transmission of white spot syndrome associated baculovirus (WSBV) in Penaeus monodon and P. japonicus via waterborne contact and oral ingestion. Aquaculture 164:263-276

Flegel TW (1997) Major viral diseases of the black tiger prawn (Penaeus monodon) in Thailand. In: Inui $\mathrm{Y}$ (ed) New approaches to viral diseases of aquatic animals. NRIA International Workshop proceedings. National Research Institute of Aquaculture, Nansei, Watarai, Mie, Japan, p $167-187$

Kim W, Abele LG (1990) Molecular phylogeny of selected decapod crustaceans based on 18s rRNA nucleotide sequences. J Crust Biol 10:1-13

Lightner DV (ed) (1996) A handbook of pathology and diagnostic procedures for diseases of penaeid shrimp. World Aquaculture Society, Baton Rouge, LA, section 3.11

Lo $\mathrm{CF}$, Leu JH, Ho $\mathrm{CH}$, Chen $\mathrm{CH}$, Peng SE, Chen YT, Chou

Editorial responsibility: Larry Vaughan,

Arlington, Massachusetts, USA
CM, Yeh PY, Huang CJ, Chou HY, Wang $\mathrm{CH}$, Kou GH (1996a) Detection of baculovirus associated with white spot syndrome (WSBV) in penaeid shrimps using polymerase chain reaction. Dis Aquat Org 25:133-141

Lo CF, Ho CH, Peng SE, Chen CH, Hsu HC, Chiu YL, Chang CF, Liu KF, Su MS, Wang CH, Kou GH (1996b) White spot syndrome baculovirus (WSBV) detected in cultured and captured shrimp, crabs and other arthropods. Dis Aquat Org 27:212-225

Lo CF, Wang CH, Kou GH (1997) White spot syndrome (WSS): pathology, hosts and prevalence in captured shrimp and crabs in Taiwan. In: Inui Y (ed) New approaches to viral diseases of aquatic animals. NRIA International Workshop proceedings. National Research Institute of Aquaculture, Nansei, Watarai, Mie, Japan, p 206-217

Wang CH, Lo CF, Leu JH, Chou CM, Yeh PY, Chou HY, Tung MC, Chang CF, Su MS, Kou GH (1995) Purification and genomic analysis of baculovirus associated with white spot syndrome (WSBV) of Penaeus monodon. Dis Aquat Org 23:239-242

Welsh J, McClelland M (1990) Fingerprinting genomes using PCR with arbitrary primers. Nucleic Acids Res 18(24):7213-7218

Williams CL, Goldson SL, Baird DB, Bullock DW (1994) Geographical origin of an introduced insect pest, Listronotus bonariensis (Kuschel), determined by RAPD analysis. Heredity 72:412-419

Williams JG, Kubelik AR, Livak KJ, Rafalski JA, Tingey SV (1990) DNA polymorphisms amplified by arbitrary primers are useful as genetic markers. Nucleic Acids Res 18(22): $6531-6535$

Williams JG, Reiter RS, Yong RM, Scolnik PA (1992) Genetic mapping of mutations using phenotypic pools and mapped RAPD markers. Nucleic Acids Res 21(11):2697-2702

Wongteerasupaya C, Vickers JE, Sriurairatana S, Nash GL, Akarajamorn A, Boonsaeng V, Panyim S, Tassanakajon A, Withyachumnarnkul B, Flegel TW (1995) A non-occluded, systemic baculovirus that occurs in cells of ectodermal and mesodermal origin and causes high mortality in the black tiger prawn Penaeus monodon. Dis Aquat Org 21:69-77

Submitted: January 15, 1998; Accepted: November 10, 1998 Proofs received from author(s): February 22, 1999 